\title{
Glucose antimetabolite 2-Deoxy-D-Glucose and its derivative as promising candidates for tackling COVID-19: Insights derived from in silico docking and molecular simulations
}

\author{
Acharya Balkrishna ${ }^{1}$, Pallavi Thakur ${ }^{2}$, Shivam Singh ${ }^{1}$, Swami Dev ${ }^{1}$, Viney Jain ${ }^{3}$, Anurag \\ Varshney $^{1}$, and Rakesh Sharma ${ }^{2}$ \\ ${ }^{1}$ Patanjali Ayurved Ltd \\ ${ }^{2}$ Affiliation not available \\ ${ }^{3}$ Jain Vishwa Bharti Institute
}

July 8, 2021

\begin{abstract}
A novel respiratory pathogen, SARS-CoV-2 has recently received worldwide attention and has been declared a public health emergency of global concern. Entry of SARS-CoV-2 is mediated through the viral spike glycoprotein (S2). Afterwards, the virus gets hold of the host cell machinery by employing the use of viral main protease 3CLpro and NSP15 endoribonuclease. In the present in silico study, active site mapping of the viral virulence factors was rendered by means of DoG Site Scorer. The possibility of repurposing of 2-deoxy-D-glucose (2-DG), a radio-chemo-modifier drug used for optimizing cancer therapy, and one of its derivative (1, 3, 4, 6-Tetra-O-acetyl-2-deoxy-D-glucopyranose, has been investigated by conducting ligand-receptor docking. Binding pose depictions of ligands and viral receptors were assessed by employing molecular dynamics analysis. Molinspiration and Toxicity Estimation Software tools were used to assess the drug likeliness, bioactivity indices and ADMETox values. 2-DG can dock efficiently with viral main protease 3CLpro as well as NSP15 endoribonuclease, thus efficiently inactivating these viral receptors leading to incapacitation of the SARS-CoV-2 virus. Such incapacitation was possible by means of formation of a hydrogen bond between 2-DG and proline residues of viral protease. The 2-DG derivative formed a hydrogen bond with the glutamine amino acid residues of the viral spike glycoprotein. The present in silico study supports the potential benefits of using 2-DG and its glucopyranose derivative as repurposed drugs/prodrugs for mitigating the novel COVID-19 infection. Since both these moieties present no signs of serious toxicity, further empirical studies on model systems and human clinical trials to ascertain effective dose-response are warranted and should be urgently initiated.
\end{abstract}

\section{Hosted file}

f4e15081a75cad2d4b380642f4e380445438.pdf available at https://authorea.com/users/307420/ articles/438392-glucose-antimetabolite-2-deoxy-d-glucose-and-its-derivative-aspromising-candidates-for-tackling-covid-19-insights-derived-from-in-silico-dockingand-molecular-simulations 


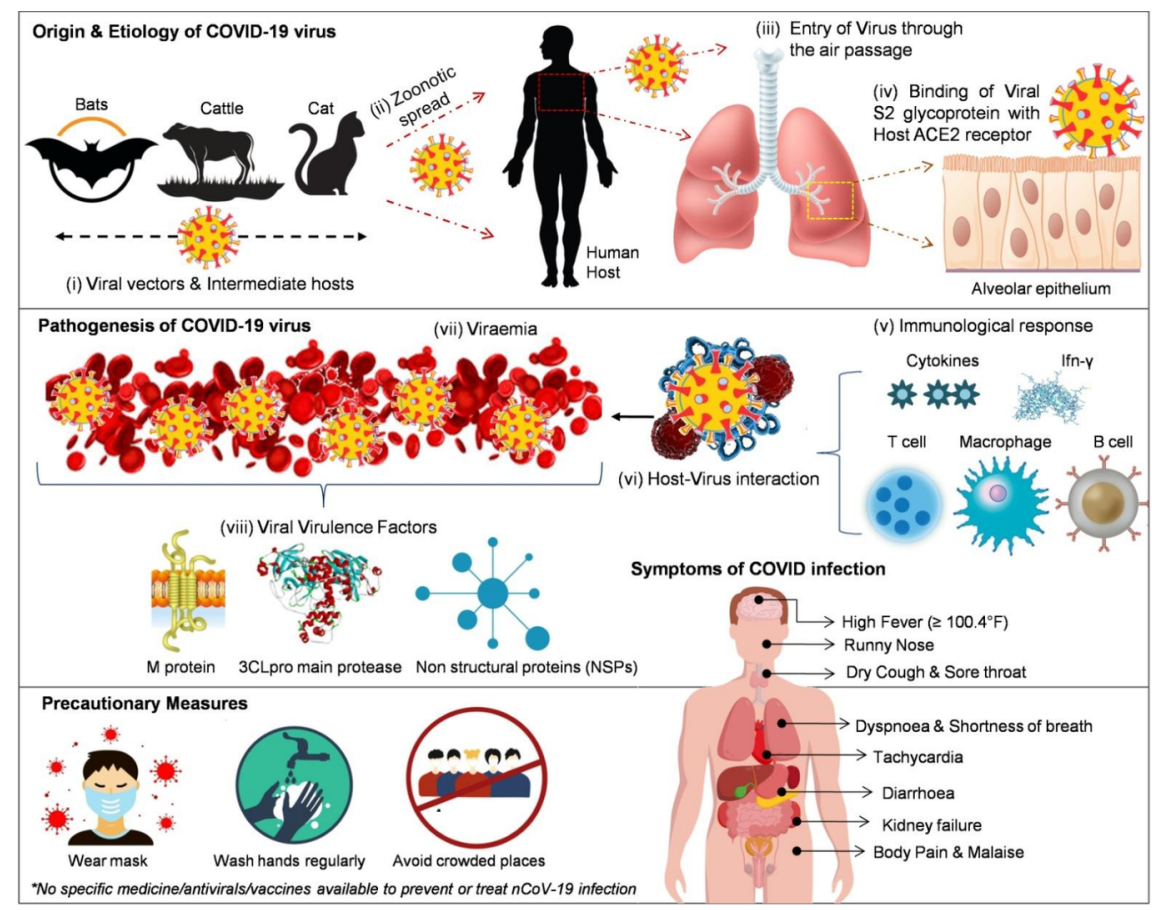

